\title{
Exploring Shopping Information and Navigation Strategies with a Mobile Device
}

\author{
David Wilfinger \\ ICT\&S Center, University of Salzburg \\ Sigmund Haffner Gasse 18 \\ 5020 Salzburg, Austria \\ david.wilfinger@sbg.ac.at
}

\author{
Astrid Weiss \\ ICT\&S Center, University of Salzbur \\ Sigmund Haffner Gasse 18 \\ 5020 Salzburg, Austria \\ astrid.weiss@sbg.ac.at
}

\author{
Manfred Tscheligi \\ ICT\&S Center, University of Salzburg \\ Sigmund Haffner Gasse 18 \\ 5020 Salzburg, Austria \\ manfred.tscheligi@sbg.ac.at
}

\begin{abstract}
In this article a field trial is presented that explores shopping information and navigation strategies and evaluates if the spectacles camera is beneficial as mobile device for this research context. The spectacles camera, a small camera installed in a pair of glasses, was used as exploration instrument in a shopping mall where passers-by could take part in a field trial on a voluntary basis. The goal of the field trial was twofold: 1.) Gaining insights on shoppers' behavior. 2.) Investigating the feasibility of the spectacles camera as exploration instrument. The field trial gave insights on navigation patterns and constituting elements of interest points of participants' shopping behavior, while the spectacles camera proved its value for investigating shopping strategies in the field.
\end{abstract}

\section{Categories and Subject Descriptors}

H.5.1.[Information Interfaces and Presentation]: Multimedia Information Systems - Evaluation/methodology

\section{General Terms}

Human Factors, Verification, Experimentation

\section{Keywords}

Navigation Strategies, Shopping Behavior, Mobile Device, Evaluation, Field Trial, Spectacles Camera.

\section{INTRODUCTION}

Recent years have shown a trend away from small corner shops towards larger shops or superstores, especially in the food retail area. Today's superstores like Walmart are not simply specialized in a certain kind of products like groceries, but additionally offer a large variety of non-food articles ranging from technical devices to school accessory. The growing size and product variety of these shops increase the difficulty for customers to find certain articles. Guidance systems, colorful poster, and trying booths are installed as a support for shoppers. Mobile technologies such as video

Copyright is held by the author/owner(s).

MobileHCI'09, September 15 - 18, 2009, Bonn, Germany.

ACM 978-1-60558-281-8. recording devices can be valuable in order to analyze navigation patterns and to evaluate existing guidance approaches without disturbing the customer while researching possibilities to support navigation and information strategies.

The presented work shows the usage of mobile technology for the exploration of shopping behavior by means of analyzing orientation strategies from a shopper's point of view. The study additionally aims at the evaluation of the spectacles camera as tool for recording the visual field of participants by assessing how well the recorded data is interpretable and how the participants perceive the overall usage and comfort of the camera. Therefore, a field trial divided into two parts was set up, with a first part aiming mainly at evaluating the participants' perception of the installed guidance system and a second part addressing the participants' main points of interest in the shop, to better understand the attributes of these points.

\section{RELATED WORK}

\subsection{The Shopping Context}

May et al. [4] argue that the last years showed a trend away from map-based orientation towards a step-by-step navigation supported approach. It is especially for the design and development of this kind of systems necessary to research human navigation strategies and patterns. Additionally, it is necessary to research, which information humans require and use for navigating in certain situations. Passini [6] shows that difficulties in orientation have a negative influence on the visitors' opinion about a room or the shop they are currently in. If shoppers get lost or if they can not navigate sufficiently, their stress level raises, which again is not positively influencing the sales. Dogu et al. [2] argue that guidance systems are especially in shopping centers supposed not only to show the way but also to support the visitors in spending a good time and having a positive shopping experience.

\subsection{Exploring Orientation Strategies}

Shopping mall behavior is already researched for example by Wesley et. al. [9], using questionnaires and interceptions for assessing decision making styles. "Unobtrusive observation methods" are used by Underhill [8] who researched costumer behaviors in retail areas of various kinds, but just from a researcher's point of view. May et al. [4] use mobile technology like a Dictaphone for researching navigation aids for pedestrians. Newcomb et al. [5] use PDAs for for supporting costumers and state the importance of investigating data on how people shop and which patterns they follow. Mobile technology for investigating way finding strategies in special contexts was already used by 
Schuchard et al. [7] who utilized a head mounted eye tracking device in order to research navigation in a nursing home. Van Herpen et al. [3] used a spectacles camera analyzing the effect of product assortment on customer choice.

\section{FIELD TRIAL}

\subsection{Goals and Research Questions}

The main goals of this field trial setup in a superstore to explore shopping orientation behavior were:

1.) To deduct design implications for indoor guidance systems in stores by exploring orientation strategies.

2.) To evaluate the usage of the spectacles camera for the purpose of analyzing navigation and orientation tasks.

The study setup was divided into two parts. Part 1 was task based, addressing shopping using a list. Part 2 was exploration based, addressing browsing shopping behavior. All participants were chosen randomly. The participants in Part 1 did not intend to enter the store, but were asked by the researchers to participate in this study whilst walking past the shop. The participants in Part 2 were asked to take part in the survey upon entering the store. The two study parts should give insights on different shopping behavior: on the perception of way finding tools, and on the suitability of the spectacles camera for contextual explorations.

Part 1 of the study included the exploration of the navigation and information strategies by assigning a task to the participants (e.g. "find frozen dumplings"):

[RQ1] How do shoppers orientate themselves in the superstore, which tools and aids do they use for orientation?

[RQ2] Which information do the shoppers use to find their way?

[RQ3] How can shoppers be better supported in their navigation tasks?

The participants in Part 2 were not given a task, but were observed during their normal shopping routine. Thus, the focus was put more on how the participants perceive the shelves and how the items in the store attracted the participants' attention and/or interest:

[RQ4] Which shopping behavior patterns can be deducted from the line of sight?

[RQ5] Which factors support the evolvement of points of interests?

Furthermore, a general research question addressed the suitability of the used mobile technology:

[RQ0] Is the spectacles camera an appropriate mobile device to evaluate shopping behavior in its context of origin?

\subsection{Instruments}

\subsubsection{Spectacles Camera}

All participants were given the spectacles camera (by Noldus Information Technologies; www.noldus.com), which is a miniature camera mounted in glasses attached to a video recording device (see figure 1).

The spectacles camera as mobile technology for exploring shopping behavior offers the following advantages:

- Cost efficiency. The acquisition costs for the spectacles camera system are significantly lower than for a mobile eye tracking system.
- Calibration. No calibration with the participants is required when using the spectacles camera, which makes it suitable for the usage in a context where people in general have little time. A long calibration phase would disturb the "real" shopping behavior.

- Children. The ease of use of the spectacles camera enabled the participation of children who we considered important for our study sample.

- Contacts/ Glasses. Data generated by the spectacles camera can not be distorted by participants wearing glasses or contact lenses.

- Less disturbing. The low weight and the simple construction similar to normal glasses reduce disturbance and the feeling of being observed.

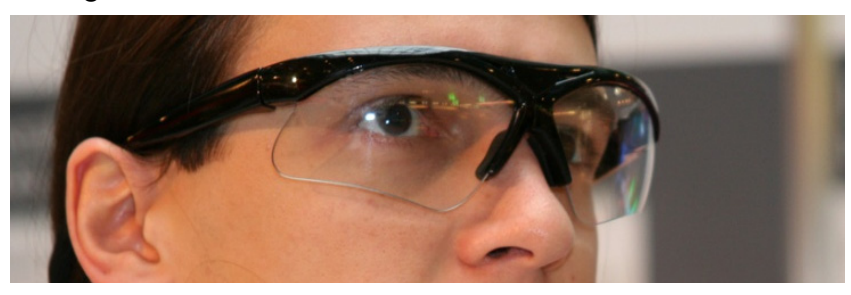

Figure 1: The Spectacles Camera

It was decided to use the spectacles camera instead of a mobile eye tracking system, despite the fact that data like mean fixation duration and gaze time in areas of interest could have resulted in more detailed insights using an eye tracking device. However, the advantages of the spectacles camera in terms of ease of use, cost efficiency, and unobtrusiveness prevailed.

\subsubsection{Think Aloud}

Shoppers were additionally introduced into the "thinking aloud" method, where participants are asked to express questions or remarks verbally. Sound was recorded by a microphone attached to the camera.

\subsubsection{Observation}

All participants were passively observed by a researcher. The researcher noted behaviors of the participant and recorded the way the shopper took through the market.

\subsubsection{Pre-Structured Interviews}

Both pre and final interviews were conducted in a pre-structured way. The pre-interview was the same for both study parts, asking for age, number of companions, frequency of visiting a store of the brand and this specific superstore, and for the properties of goods that attract participants in their opinion.

The final interview for both study parts contained questions about orientation in the store, information that helped, and what information was missing. All final interviews concluded with questions on the spectacles camera, if it disturbed the participants, if they felt observed, and if they felt influenced by the camera when walking through the shop.

\subsubsection{Video Analysis}

For the purpose of later interpretation the video taken by the spectacles camera was saved on a hardware recorder that the participants carried along in a belt bag.

\subsubsection{Data Analysis}

All the qualitative data gathered in this case study (think aloud, observation, and video analysis) was analyzed in an iterative 
spiral [1] including the following steps: (1) Managing data and finding a focus, (2) categorizing data, linking data and connecting categories, (3) producing an account. The categorization of the data focused on orientation strategies, behavioral patterns, and points of interest. The produced account in terms of quantifications and descriptive shopping patterns will be presented in the following chapter.

\section{RESULTS}

10 men and 8 women participated in the study; they were from 7 to 59 years old with 28.56 years on average (SD 18.79). 6 shoppers participated in part 2 of the study as they had plans to shop in the superstore, the other 12 participants were sent into the store with the task to find a certain product (study part 1). 14 participants reported that they shop at least once a week in a store of that chain while 7 shoppers stated that they would go at least once a week into that specific store. For the shoppers the products were interesting because of habit (10), price (9), and curiosity (8) with multiple answers possible.

\subsection{Shoppers' Orientation - Tools [RQ1]}

The interviews revealed that shoppers orientate themselves using knowledge gained earlier in the same or in similar stores (9). Experience and habits play a major role (8). The guidance system was used to find similar products or product areas, where customers knew by experience or simply assumed that the desired item was located.

According to the spectacles camera's video and audio material, the participants navigate by mentally scaling the shop areas, meaning that shoppers orientate themselves more detailed the closer they come to the item they are looking for. When looking for frozen sweet dumplings the participants first tried to find the frozen food department and then the desserts before really looking for the dumplings. Therefore, the participants mentally divided the shop into certain areas that were again subdivided until they had found what they were looking for, an insight that was proven by the analyses of stopping points and the path of the shoppers recorded by the observing researcher.

\subsection{Shoppers' Orientation - Information [RQ2]}

In the final interview the participants of part 1 of the study (task: find a specific product) reported that they were mainly using types of goods in the shelves and certain shop areas (11) for orientation purposes, own experience (5) was also mentioned as tool for finding their way, and signs (5) were as well reported to be helpful (multiple answers possible). No shopper complained about missing information. Some participants for example requested larger fonts on signs, more and better labeling of item groups, and direction arrows on special offer signs. No participant was able to remember the labeling of the shelf with the product that he or she was looking for.

The participants in the second part of the study who had already planned to visit the superstore were mainly finding their way by orientating after their previous experience in this shop or shops of the same brand with similar layout (5). One participant stated: "I know this shop, they all look the same". Three participants stated that they use similar products for orientation and noted, that through their experience they would know the area of certain types of goods they were looking for in the shop. Shoppers stated that a different color layout (e.g. floor, shelves, walls) of the single areas would support their orientation.
The in-depth analyses of the video and audio material recorded with the spectacles camera confirmed the statements of the users given in the final interview. A main source of information for orientation was the installed guidance system (10). The participants were standing in the shop, turning and browsing articles until they had found a group of products or a certain shelf that they associated with the item they were looking for (7).

However, it was stated in the final interviews that not only the mounted signs were used as a guidance system, but also previous knowledge of the store gained earlier in time and the shoppers' opinion about how shelves should be arranged (5) were used for finding the way. Only two participants used other information sources like a companion or panels.

\subsection{Support for Navigation Tasks [RQ3]}

Both, video and observational data indicated that navigation strategies are mainly product oriented (see RQ2), meaning that shoppers used properties of products in the shelves to find what they were looking for. A major improvement for guidance systems therefore would be the combination of text with images or icons on the signature of the guidance system. This would allow the shoppers to scan the signs without reading them. Additionally, it is recommended that shops indicate their individual product departments and the borders between them clearer than it is done so far in the explored superstore.

According to the final interview, the customers stated that they also use their experience and their shopping habits for orientation (e.g. ,vegetables are always in the front left section”). Therefore, it is recommended that products and product groups stay arranged similarly in the shop even if for example special offers are placed in the store. These special offers could lead to confusion when customers recognize them and assume that more similar products can be found in the same spot.

\subsection{Shopping Behavior Patterns [RQ4]}

Based on the interpretation of the spectacles camera's video and the interview material of the 18 participants, it was possible to deduct four main behavior patterns people develop while shopping in the superstore.

Ambient Shopper: The Ambient Shopper takes his/her time and decides which items to buy while shopping. It is a main purchasing criterion if products are placed in the surrounding area. All Ambient Shoppers were identified in the group of participants that had already intended to visit the store and had no task to fulfill, which explains this result.

Hunter: Hunters were with one exception all participants that performed the task of finding a certain item in the store. Hunters perform area related shopping, meaning that they directly walk to the expected area of the searched item and then browse the surrounding shelves looking for their catch to make.

Observer: Observers examine their surrounding and browse through all products. Their shopping is product related. Observers were found in both parts of the study.

Speedshopper: Speedshoppers are very experienced shoppers who have their standard shopping route. Efficiency rules their shopping activity. This behavior pattern was found in the second part of the study.

Important to note in this context is that shopping strategies changed within the shopping activity and that some shoppers used 
more than one strategy depending on the situation and the appropriateness of the method.

\subsection{Points of Interest [RQ5]}

Based on the analysis of the video captured by the spectacles camera showing besides others the view direction of the shoppers, several factors and items were identified that supported the evolvement of interest points. Examples for those are large or free hanging special offer signs (5) and backlit signs like the ones mounted over bread or meat sections (5). Such points were also generated by products that were apparently out of place and by goods that were of a special interest for the shoppers (e.g. the favorite magazines in a shelf) (1). A signature, detached from the shelves, therefore seems to be attracting a shopper's attention.

\subsection{The Spectacles Camera as Exploration Tool [RQ0]}

Based on the results presented in the sections 4.1 to 4.5 , the appropriateness of the spectacles camera for the methodological setup was evaluated. The results prove the value of the spectacles camera as mobile device to study shoppers' information and navigation strategies.

1. Value and richness of the data: The spectacles camera has proven its value to investigate navigation strategies directly in the relevant context and from the participants' view. This enabled us to develop a holistic picture of the navigation and information strategies by combining internal (spectacles camera) and external (researcher observation) behavioral data with reflective interview material. By that all proposed research questions [RQ1] - [RQ5] could be answered.

2. Methodological support: The methodological set-up provided a low-intrusion variation of participant-controlled observation. The participants of the study did not have to think about recording important events (like e.g. with a portable hand-held camera). Furthermore, the spectacles camera simply recorded everything in the shoppers' field of view, and thus produced objective behavioral data.

3. Participant assessment: In the final interview only three participants stated that the camera disturbed them on their way through the superstore. Five participants stated that they felt observed on their way through the shop; one participant reported that this was mainly due to the microphone and the sound recording. Additionally, three shoppers explained that they felt influenced on their way through the store because of the camera.

One negative issue of using the spectacles camera was that the video recorder was not able to stream the video signal wireless, which would be a great advantage for future field trials.

\section{LESSONS LEARNED AND NEXT STEPS}

Considering our experiences we recognized the following issues as being crucial to successfully evaluated navigation strategies by means of the spectacles camera:

1. Participant sampling: When conducting a study in the field with the spectacles camera it is possible to recruit a diverse sample on-site, as the camera can be used by children, adults, and elderly people without much explanation or calibration.

2. Method mix: It is recommended to conduct a spectacles camera field trial with an accompanying pre-structured interview and observational data. Linking the spectacles camera videos with the think aloud data facilitated the analysis.

3. Limited generalization: Investigating a specific superstore brand does not allow generalizing results in terms of shopping behavior. Several further tests will be necessary to achieve an understanding of shoppers' navigation and information strategies in general.

To gain more insights on general navigation and information strategies in the shopping context follow-up field trials are planned with more participants in several stores and shopping situations with different underlying navigation and orientation patterns to gain results that can be generalized. Furthermore, live streaming of the video will give researchers but also members of marketing and the industry the possibility to conduct the external observation without shadowing the participants of the study and react to events in the final interview.

\section{ACKNOWLEDGMENTS}

The authors want to thank all participants, Martin Altmanninger, Michael Pirker, Stefan Riegler, Daniel Schwaiger, and INTERSPAR for their support in the study.

\section{REFERENCES}

[1] Dey, I. 1993. Qualitative data analysis: A user-friendly guide for social scientists. London: Routledge.

[2] Dogu, U. and Erkip, F. 2000. Spatial Factors Affecting Wayfinding and Orientation: A Case Study in a Shopping Mall. Environment and Behavior, 32, 6 (November 1, 2000), 731-755.

[3] Herpen van, E.; van Trijp, H.; Kuipers, T. (2005). The influence of assortment organization on product comparisons and choice. Proceedings of the 5th International Conference on Methods and Techniques in Behavioral Research, pp. 595-596.

[4] May, A. J., Ross, T., Bayer, S. H. and Tarkiainen, M. J. 2003. Pedestrian navigation aids: information requirements and design implications. Personal Ubiquitous Comput., 7, 6 2003), 331-338.

[5] Newcomb, E., Pashley, T. and Stasko, J. 2003. Mobile computing in the retail arena. Proceedings of the conference on Human factors in computing systems CHI2003, ACM.

[6] Passini, R. 1996. Wayfinding design: logic, application and some thoughts on universality. Design Studies, 17, 3 1996, 319-331.

[7] Schuchard, R., Connell, B., and Griffiths, P. 2006. An environmental investigation of wayfinding in a nursing home. Proceedings of the 2006 Symposium on Eye Tracking Research \& Applications. ETRA '06. ACM, NY

[8] Underhill, P. 1999. Why We Buy: The Science of Shopping. Simon \& Schuster Press, NY

[9] Wesley, S., LeHew, M. and Woodside, A. G. 2006. Consumer decision-making styles and mall shopping behavior: Building theory using exploratory data analysis and the comparative method. Journal of Business Research, 59, 5 (2006), 535-548. 